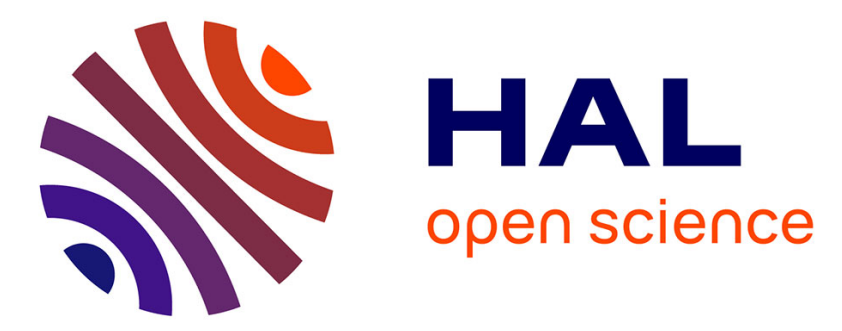

\title{
Finite-Element Modeling of Thermoelastic Attenuation in Piezoelectric Surface Acoustic Wave Devices
}

\author{
Zhi Qin, Hakeim Talleb, Jean-Yves Duquesne, Massimiliano Marangolo, \\ Zhuoxiang Ren
}

\section{- To cite this version:}

Zhi Qin, Hakeim Talleb, Jean-Yves Duquesne, Massimiliano Marangolo, Zhuoxiang Ren. FiniteElement Modeling of Thermoelastic Attenuation in Piezoelectric Surface Acoustic Wave Devices. IEEE Transactions on Magnetics, 2015, 51 (3), pp.7401904 10.1109/TMAG.2014.2361401 • hal-01149020

\section{HAL Id: hal-01149020 https: / hal.sorbonne-universite.fr/hal-01149020}

Submitted on 8 Apr 2016

HAL is a multi-disciplinary open access archive for the deposit and dissemination of scientific research documents, whether they are published or not. The documents may come from teaching and research institutions in France or abroad, or from public or private research centers.
L'archive ouverte pluridisciplinaire HAL, est destinée au dépôt et à la diffusion de documents scientifiques de niveau recherche, publiés ou non, émanant des établissements d'enseignement et de recherche français ou étrangers, des laboratoires publics ou privés. 


\title{
Finite-Element Modeling of Thermoelastic Attenuation in Piezoelectric Surface Acoustic Wave Devices
}

\author{
Zhi Qin ${ }^{1}$, Hakeim Talleb ${ }^{1}$, Jean-Yves Duquesne ${ }^{2}$, Massimiliano Marangolo ${ }^{2}$, and Zhuoxiang Ren ${ }^{1}$ \\ ${ }^{1}$ Sorbonne Universités, UPMC Univ. Paris 06, UR2, L2E, F-75005 Paris, France \\ ${ }^{2}$ CNRS, UMR7588, Institut des Nanosciences de Paris (INSP), F-75005, Paris, France, \\ Sorbonne Universités, UPMC Univ. Paris 06, UMR7588, INSP Paris F-75005, Paris, France
}

\begin{abstract}
The assessment of thermoelastic attenuation is crucial in designing surface acoustic wave (SAW) devices. As irregular structures are more and more involved in modern applications for which efficient numerical tools are required, a multi-physics finite-element model is proposed in this paper, where thermoelastic damping in piezoelectric materials can be accounted for in both coated and uncoated conditions. The coupled equations are solved iteratively in time domain, using the Newmark method. The mechanical, electrical, and thermal degrees of freedom are calculated simultaneously at each time step. An application of the model is presented through the investigation of thermoelastic loss in a lithium niobate SAW device.
\end{abstract}

Index Terms - Electrothermoelastic coupling, multi-physics finite-element (M-FE) analysis, piezoelectric materials, thermoelastic effect.

\section{INTRODUCTION}

$\mathbf{S}$ URFACE ACOUSTIC WAVE (SAW) devices are widely used due to the small size, high reliability, and signal processing capability. For instance, SAWs are employed for microparticle separation [1] in flow-based analysis. The SAW-based sensors in biomedical [2] and space research [3] are also broadly used.

A SAW device usually consists of a piezoelectric substrate and deposited interdigital transducers (IDTs). The IDTs can be divided into transmitting and receiving pairs. The SAW can be stimulated when electric signals are applied to the transmitting IDTs. They can then be measured when reaching the receiving IDTs. The part between both pairs is the delay-line area, where sensitive films or fluidic parts can be integrated. Since most of the energy is concentrated near the surface, even very tiny variation in the delay-line area can be detected, which makes the SAW device ideal for sensing and micromanipulation. The operating frequencies are normally located in megahertz range and the thermoelastic attenuation becomes significant [4]. This loss is, in fact, due to cyclic heat flow from the region of compressive stress to the region of tensile stress. Elastic energy is thus partly converted into heat and dissipated.

The mechanism of thermoelastic attenuation of SAW were descript in isotropic media [5] and layered structures [6]. Analytical models for quantitative calculation were developed in [7], on the basis of perturbation theory. Even though analytically calculated thermoelastic loss coincided well with the experimental results in simple cases, the developments and assumptions were somehow cumbersome and can be invalid in other cases. For example, microcavities in the substrate are frequently shown in flow-based analysis [1], which makes the homogeneous half space assumption of the substrate no longer hold. The finite-element method, on the other hand, is versatile due to its flexibility in modeling complicated geometry and its capability in obtaining fully coupled multi-physics field solutions. Coupled electrothermoelastic finite-element models have been built in preceding works [8], [9]. However, these models were mostly for thermal shock problems, thermoelastic damping problems remain to be investigated. Here, we developed a new multi-physics finite-element (M-FE) model dedicating for thermoelastic attenuation in SAW devices. This paper is organized as follows. Governing equations of thermoelastic attenuation in piezoelectric materials and their finite-element formulations are presented in Section II. It is followed by a numerical example of the developed M-FE model applied to the investigation of thermoelastic attenuation in a lithium niobate substrate in both coated and uncoated cases. Finally, the conclusions are drawn in Section IV.

\section{Multi-Physics EQuations AND FINITE-ELEMENT FORMULATIONS}

\section{A. Multi-Physics Equations}

In what follows, we use the Einstein summation convention to describe different physic equations. Linear theory of thermopiezoelectricity can be found in [9] among others. Primary procedures are adapted and resumed in this section. In the absence of body force, free charge and internal heat generation, the equilibrium relations of the coupled fields can be expressed in (1), where $\sigma_{i j}$ is the vector of stress tensor, $u_{i}$ vector of elastic displacement in the ith direction, $\rho$ mass density, $q_{i}$ vector of heat flux, $T_{0}$ initial equilibrium temperature, $\eta$ entropy density, and $D_{i}$ vector of electric displacement. A comma subscript followed by an index number $i$ indicates a derivation with respect to the corresponding coordinate direction $i$

$$
\begin{aligned}
\sigma_{i j, j} & =\rho \ddot{u}_{i} \\
q_{i, i} & =-T_{0} \rho \dot{\eta} \\
D_{i, i} & =0 .
\end{aligned}
$$

By noting with $\varepsilon_{i j}$ indicating the vector of strain tensor, $E_{i}$ vector of electric intensity, $\phi$ electric potential, $k_{i j}$ tensor 
of heat conduction coefficients, and $\theta=T-T_{0}$ difference between actual and initial temperatures, linear comportments of elastic, electric, and thermal fields read as follows:

$$
\begin{aligned}
\varepsilon_{i j} & =\left(u_{i, j}+u_{j, i}\right) / 2 \\
E_{i} & =-\phi_{, i} \\
q_{i} & =-k_{i j} \theta_{, j} .
\end{aligned}
$$

The constitutive equations can then be expressed by

$$
\begin{aligned}
\sigma_{i j} & =c_{i j k l} \varepsilon_{k l}+e_{k i j} \phi_{, k}-\alpha_{i j} \theta \\
D_{i} & =e_{i k l} \varepsilon_{k l}-p_{i k} \phi_{, k}+d_{i} \theta \\
\rho \eta & =\alpha_{k l} \varepsilon_{k l}-d_{k} \phi_{, k}+c_{E} \theta
\end{aligned}
$$

where $c_{i j k l}$ is the stiffness tensor, $e_{i k l}$ piezoelectric tensor, $\alpha_{i j}$ tensor of thermal stress coefficients, $p_{i k}$ permittivity tensor, $d_{i}$ vector of pyroelectric coefficients, and $c_{E}$ the specific heat per unit volume at constant deformation (more detailed definitions of the quantities can be found in [10]). Substitution of (2) and (3) in (1) yields the governing equations, which take the form

$$
\begin{aligned}
\rho \ddot{u}_{i} & =c_{i j k l} u_{k, l j}+e_{k i j} \phi_{, k j}-\alpha_{i j} \theta_{, j} \\
0 & =e_{i k l} u_{k, l i}-p_{i k} \phi_{, k i}+d_{i} \theta_{, i} \\
k_{i j} \theta_{, i j} & =T_{0}\left(\alpha_{i j} \dot{u}_{i, j}-T_{0} d_{i} \dot{\phi}_{, i}+c_{E} \dot{\theta}\right) .
\end{aligned}
$$

For multi-physics investigations with (4) in a domain $\Omega$, the boundary conditions along its boundary $\partial \Omega$ should be satisfied. The continuity of $u_{i}, \phi$, and the normal component of $D_{i}, \sigma_{i j} n_{j}$, and $k_{i j} T,{ }_{j} n_{j}$ ( $n_{j}$ is the normal unit vector) at an interface have to be fulfilled.

\section{B. Finite-Element Formulations}

The variational principle is applied to obtain weak forms. More specifically, equations in (4) are multiplied with the test functions $u_{i}^{\prime}, \phi^{\prime}$, and $\theta^{\prime}$, and integrated by part, respectively. The following equations, in considering corresponding boundary conditions represent the variational form:

$$
\begin{gathered}
\int_{\Omega} u_{i, j}^{\prime}\left(c_{i j k l} u_{k, l}+e_{k i j} \phi_{, k}-\alpha_{i j} \theta\right) d V+\int_{\Omega} u_{i}^{\prime} \rho \ddot{u}_{i} d V=0 \\
\int_{\Omega} \phi_{,{ }_{i}}\left(e_{i k l} u_{k, l}-p_{i k} \phi_{, k}+d_{i} \theta\right) d V=0 \\
\int_{\Omega} \theta^{\prime}\left(T_{0} \alpha_{i j} \dot{u}_{i, j}-T_{0} d_{i} \dot{\phi},{ }_{i}+c_{E} \rho \dot{\theta}\right) d V+\int_{\Omega} \theta_{,{ }_{i}} k_{i j} \theta,{ }_{j} d V=0 .
\end{gathered}
$$

They are spatially discredited by interpolating the field unknowns in an element $\boldsymbol{u}_{e}, \phi_{e}$, and $\theta_{e}$ in terms of nodal degrees of freedom (DoFs) $\boldsymbol{u}_{k}, \boldsymbol{\phi}_{k}$, and $\boldsymbol{\theta}_{k}$ using appropriate nodal shape functions $N_{u}, N_{\phi}$, and $N_{\theta}$, as shown in

$$
\boldsymbol{u}_{e}=\boldsymbol{N}_{u} \boldsymbol{u}_{k} \quad \phi_{e}=\boldsymbol{N}_{\phi} \boldsymbol{\phi}_{k} \quad \theta_{e}=\boldsymbol{N}_{\theta} \boldsymbol{\theta}_{k}
$$

For brevity, the following differential matrices are defined:

$$
\begin{gathered}
D_{u}=\left[\begin{array}{ccc}
\partial / \partial x_{1} & 0 & 0 \\
0 & \partial / \partial x_{2} & 0 \\
0 & 0 & \partial / \partial x_{3} \\
\partial / \partial x_{1} & \partial / \partial x_{2} & 0 \\
0 & \partial / \partial x_{2} & \partial / \partial x_{3} \\
\partial / \partial x_{1} & 0 & \partial / \partial x_{3}
\end{array}\right] \\
D_{\phi}=D_{\theta}=\left[\begin{array}{l}
\partial / \partial x_{1} \\
\partial / \partial x_{2} \\
\partial / \partial x_{3}
\end{array}\right] .
\end{gathered}
$$

Then, differentials of shape functions can be denoted as

$$
\boldsymbol{B}_{u}=D_{u} \boldsymbol{N}_{u} \quad \boldsymbol{B}_{\phi}=D_{\phi} \boldsymbol{N}_{\phi} \quad \boldsymbol{B}_{\theta}=D_{\theta} \boldsymbol{N}_{\theta} .
$$

Substituting (6) and (8) into (5) leads to element level equations

$$
\begin{array}{r}
\boldsymbol{M}_{u u}^{e} \ddot{\boldsymbol{u}}_{k}+\boldsymbol{K}_{u u}^{e} \boldsymbol{u}_{k}+\boldsymbol{K}_{u \phi}^{e} \boldsymbol{\phi}_{k}-\boldsymbol{K}_{u \theta}^{e} \boldsymbol{\theta}_{k}=\boldsymbol{f}_{u}^{e} \\
\boldsymbol{K}_{\phi u}^{e} \boldsymbol{u}_{k}-\boldsymbol{K}_{\phi \phi}^{e} \boldsymbol{\phi}_{k}+\boldsymbol{K}_{\phi \theta}^{e} \boldsymbol{\theta}_{k}=\boldsymbol{f}_{\phi}^{e} \\
\boldsymbol{K}_{\theta u}^{e} \dot{\boldsymbol{u}}_{k}-\boldsymbol{K}_{\theta \phi}^{e} \dot{\boldsymbol{\phi}}_{k}+\boldsymbol{H}_{\theta \theta}^{e} \dot{\boldsymbol{\theta}}_{k}+\boldsymbol{K}_{\theta \theta}^{e} \boldsymbol{\theta}_{k}=\boldsymbol{f}_{\theta}^{e}
\end{array}
$$

where

$$
\begin{aligned}
& \boldsymbol{M}_{u u}^{e}=\int_{\Omega}^{e} \boldsymbol{N}_{u}^{T} \rho \boldsymbol{N}_{u} d \Omega \quad \boldsymbol{K}_{u u}^{e}=\int_{\Omega}^{e} \boldsymbol{B}_{u}^{T} \boldsymbol{C} \boldsymbol{B}_{u} d \Omega \\
& \boldsymbol{K}_{u \phi}^{e}=\int_{\Omega}^{e} \boldsymbol{B}_{u}^{T} e^{T} \boldsymbol{B}_{\phi} d \Omega \quad \boldsymbol{K}_{u \theta}^{e}=\int_{\Omega}^{e} \boldsymbol{B}_{u}^{T} \boldsymbol{\alpha} \boldsymbol{N}_{\theta} d \Omega \\
& \boldsymbol{K}_{\phi u}^{e}=\int_{\Omega}^{e} \boldsymbol{B}_{\phi}^{T} \boldsymbol{e} \boldsymbol{B}_{u} d \Omega \quad \boldsymbol{K}_{\phi \phi}^{e}=\int_{\Omega}^{e} \boldsymbol{B}_{\phi}^{T} \boldsymbol{p} \boldsymbol{B}_{\phi} d \Omega \\
& \boldsymbol{K}_{\phi \theta}^{e}=\int_{\Omega}^{e} \boldsymbol{B}_{\phi}^{T} d \boldsymbol{B}_{\theta} d \Omega \quad \boldsymbol{K}_{\theta u}^{e}=\int_{\Omega}^{e} T_{0} \boldsymbol{N}_{u}^{T} \boldsymbol{\alpha}^{T} \boldsymbol{B}_{u} d \Omega \\
& \boldsymbol{K}_{\theta \phi}^{e}=\int_{\Omega}^{e} T_{0} \boldsymbol{N}_{u}^{T} \boldsymbol{d}^{T} \boldsymbol{B}_{\theta} d \Omega \quad \boldsymbol{K}_{\theta \theta}^{e}=\int_{\Omega}^{e} \boldsymbol{B}_{\theta}^{T} \boldsymbol{k} \boldsymbol{B}_{\theta} d \Omega \\
& \boldsymbol{H}_{\theta \theta}^{e}=\int_{\Omega}^{e} \boldsymbol{N}_{\theta}^{T} \rho c_{E} \boldsymbol{N}_{\theta} d \Omega .
\end{aligned}
$$

Element-level matrices are then assembled to form the global equations, as presented in

$$
\begin{gathered}
{\left[\begin{array}{ccc}
\boldsymbol{M}_{u u} & \mathbf{0} & \mathbf{0} \\
\mathbf{0} & \mathbf{0} & \mathbf{0} \\
\mathbf{0} & \mathbf{0} & \mathbf{0}
\end{array}\right]\left[\begin{array}{l}
\ddot{\boldsymbol{u}} \\
\ddot{\boldsymbol{\phi}} \\
\ddot{\boldsymbol{\theta}}
\end{array}\right]+\left[\begin{array}{ccc}
\mathbf{0} & \mathbf{0} & \mathbf{0} \\
\mathbf{0} & \mathbf{0} & \mathbf{0} \\
\boldsymbol{K}_{\theta u} & -\boldsymbol{K}_{\theta \phi} & \boldsymbol{H}_{\theta \theta}
\end{array}\right]\left[\begin{array}{c}
\dot{\boldsymbol{u}} \\
\dot{\boldsymbol{\phi}} \\
\dot{\boldsymbol{\theta}}
\end{array}\right]} \\
+\left[\begin{array}{ccc}
\boldsymbol{K}_{u u} & \boldsymbol{K}_{u \phi} & -\boldsymbol{K}_{u \theta} \\
\boldsymbol{K}_{\phi u} & -\boldsymbol{K}_{\phi \phi} & \boldsymbol{K}_{\phi \theta} \\
\mathbf{0} & \mathbf{0} & \boldsymbol{K}_{\theta \theta}
\end{array}\right]\left[\begin{array}{l}
\boldsymbol{u} \\
\boldsymbol{\phi} \\
\boldsymbol{\theta}
\end{array}\right]=\left[\begin{array}{l}
\boldsymbol{f}_{u} \\
\boldsymbol{f}_{\boldsymbol{\phi}} \\
\boldsymbol{f}_{\boldsymbol{\theta}}
\end{array}\right] .
\end{gathered}
$$

Since the unknown DoFs are of different orders of magnitude, it is numerically convenient to treat them as non-dimensional quantities (the asterisk symbol marked as follows) by introducing the following relations [8]:

$$
\begin{aligned}
& x_{i}^{*}=c_{0} \xi_{0} x_{i}, \quad u_{i}^{*}=c_{0} \xi_{0} u_{i}, \quad t^{*}=c_{0}^{2} \xi_{0} t \\
& \phi^{*}=c_{0}^{2} \xi_{0} \delta_{0} \phi, \quad \theta^{*}=\theta / T_{0}, \quad c_{0}=\sqrt{c_{33} / \rho} \\
& \xi_{0}=\rho c_{E} / k, \quad \delta_{0}=e_{33} / c_{33}
\end{aligned}
$$




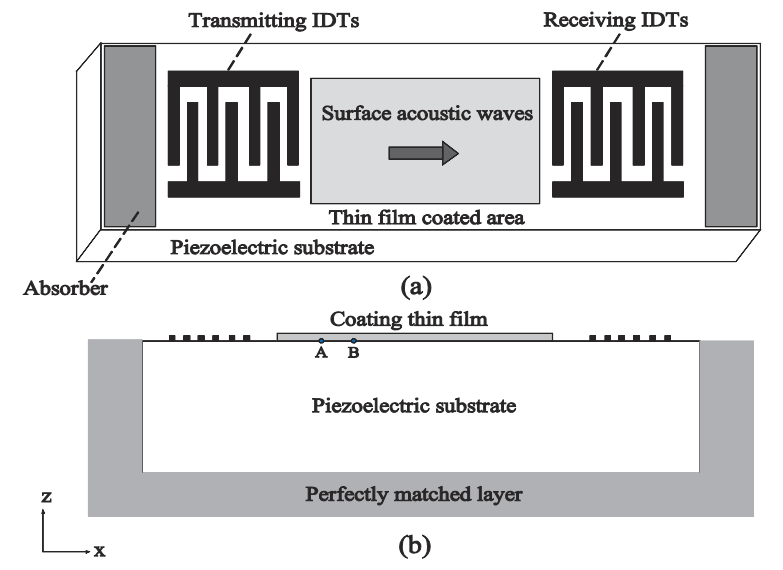

Fig. 1. (a) Typical SAW device schema [14]. (b) 2-D model configuration.

where $c_{33}$ and $e_{33}$ are corresponding components in the stiffness and piezoelectric tensor, respectively, $k$ the heat conduction coefficients, and $c_{0}$ the propagating velocity of elastic wave in the solid. Calculated results are converted back to the original quantities for their visibility of physics sense. Equation (11) is integrated in time domain using the Newmark method, being unconditionally stable and of secondorder accuracy.

\section{Absorbing Boundary Conditions}

An important issue worthy noting here is the absorbing boundary condition, i.e., the perfectly matched layer (PML), with which one can avoid wave reflections. Otherwise, larger dimensions need to be constructed wasting too much computing resource. Demonstration of PML in modeling SAW devices in frequency and time domain can be found in [11] and [12], respectively. Time domain implementation is rather complicated due to the frequency dependent nature of some parameters in PML setting. The memory variables [13] are used in the work to avoid convolution terms resulted from the inverse Fourier transform of frequency domain formulations. A three wavelength wide PML boundary is set to sides of the computing domain except for the free surface (as presented in Section III). Reflections are thus successfully avoided; the demand for computing resources is also significantly reduced.

\section{NUMERICAL EXAMPLES}

\section{A. Modeling of SAWs in Piezoelectric Substrate}

The M-FE model is evaluated through modeling of the Rayleigh SAWs propagating in the $x$-direction and $z$-direction in a $Y$-cut $\mathrm{LiNbO}_{3}$ substrate. A similar configuration [Fig. 1(a)] has been adapted from [14].

Five volts peak-to-peak sinusoidal electric signals of central frequency $f_{0}=300 \mathrm{MHz}$ are applied to the transmitting IDTs. Corresponding analytical solutions of displacements and potential take the form of (13), in which $\alpha=1,2,3$; $b_{n}$ indicates the decaying coefficient along $z$-direction when no other attenuations are accounted for, $U_{i}^{n}$ and $\Phi_{i}^{n}$ are initial values, $k$ the wave number, and $v$ the wave velocity [15]. As (13) indicates, components in $y$-direction are decoupled. This enables a model in 2-D possible. Fig. 1(b) shows the
TABLE I

Material Constants of the $Y$-CUt Lithium Niobate

\begin{tabular}{|c|c|c|c|}
\hline \multirow[t]{2}{*}{ Elastic moduli (GPa) } & $\mathrm{c}_{11}=203$ & $c_{33}=245$ & $\overline{c_{12}=53}$ \\
\hline & $c_{44}=60$ & $c_{13}=75$ & $c_{14}=9$ \\
\hline \multirow[t]{2}{*}{ Piezoelectric const. $\left(\mathrm{C} \cdot \mathrm{m}^{-2}\right)$} & $e_{15}=3.7$ & $e_{31}=0.2$ & \\
\hline & $e_{22}=2.5$ & $e_{33}=1.3$ & \\
\hline Dielectric const. $\left(10^{-12} F \cdot m^{-1}\right)$ & $\varepsilon_{11}=389$ & $\varepsilon_{33}=257$ & \\
\hline $\begin{array}{l}\text { Thermal expansion coeff. } \\
\left(10^{-5} K^{-1}\right)\end{array}$ & $\alpha_{11}=1.5$ & $\alpha_{33}=0.5$ & \\
\hline $\begin{array}{l}\text { Pyroelectric coeff. } \\
\left(10^{-5} \mathrm{CK}^{-1} \mathrm{~m}^{-2}\right)\end{array}$ & $P_{z}^{\prime}=-4$ & & \\
\hline $\begin{array}{l}\text { Thermal conductivities } \\
\left(\mathrm{Jm}^{-1} \mathrm{~s}^{-1} \mathrm{~K}^{-1}\right)\end{array}$ & $k_{1}=18.5$ & $k_{3}=18.5$ & \\
\hline Specific heat $\left(10^{6} \mathrm{Jm}^{-3} \mathrm{~K}^{-1}\right)$ & $c_{E}=4.18$ & & \\
\hline Mass density $\left(\mathrm{kg} \cdot \mathrm{m}^{-3}\right)$ & $\rho=4700$ & & \\
\hline $\begin{array}{l}\text { Rayleigh wave velocity } \\
\left(\mathrm{m} \cdot \mathrm{s}^{-1}\right)\end{array}$ & $v_{0}=3488$ & & \\
\hline
\end{tabular}

2-D model in which PML is applied

$$
\begin{aligned}
& u_{\alpha}=\sum_{n=1}^{2} U_{i}^{n} \exp \left(k b_{j} z\right) \exp (i k(x-v t)) \\
& \phi_{\alpha}=\sum_{n=1}^{2} \Phi_{i}^{n} \exp \left(k b_{j} z\right) \exp (i k(x-v t)) .
\end{aligned}
$$

A program was developed in MATLAB in which a substrate of $30 \lambda$ wide and $10 \lambda$ high ( $\lambda$ is the wavelength) was built. 24 electrodes were modeled in transmitting and receiving IDTs, which are separated by $20 \lambda$ and of period of $\lambda$. In the coated situation, the thin film was set to be $1 / 100 \lambda$ and assumed to be isotropic and electrically non-conductive. Delaunay triangular elements were employed with maximal element length set to be $1 / 32 \lambda$, and the time step set to $1 / 40$ $T^{0}$ with $T^{0}=1 / f_{0}$ being the signal period. Electrically grounded and mechanically fixed conditions have been set to the bottom to reduce the second-order effects of the piezoelectric effect [14]. For nodes on the upper surface on which electrodes are supposed to be located, electric potentials are explicitly defined as boundary conditions. In the coated case, continuity of displacement and temperature fields is demanded at the interface. Besides, the surface of the coating material can be regarded as isothermal [4] and electrically non-conductive. Hence, the corresponding thermal and electric DoFs need to be set as identical and ignored, respectively.

Material constants of the substrate are referred to [1] and summarized in Table I. Elastic and thermoelastic constants are set to be identical to, while the specific heat and thermal conductivity of the film to be half of the corresponding values of the substrate.

\section{B. Results and Discussion}

A contour plot of displacements in the normal direction is shown in Fig. 2. As can be seen, the wave intensity dies down in the normal direction till null at about three wavelengths away from the surface. In Fig. 3, displacements in the normal direction of points A and B [Fig. 1(b)] are shown. Point A is closer to the stimulating source and locates four wavelengths away from point B. Displacements at both points oscillate at constant amplitudes after sometime. Due to the presence of thermoelastic damping, slight descending in the amplitude in B can be observed. Figs. 2 and 3 qualitatively verified the propagating characteristics of SAW as described by (13).

A quantitatively validation can be found in Fig. 4 in which comparisons between the results of numerical and analytical 


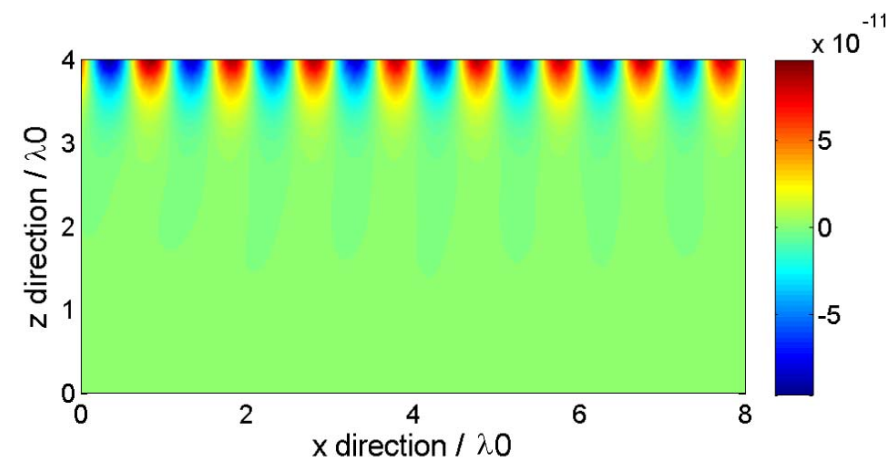

Fig. 2. Contour plot of displacements in $z$-direction at $15 T_{0}$.

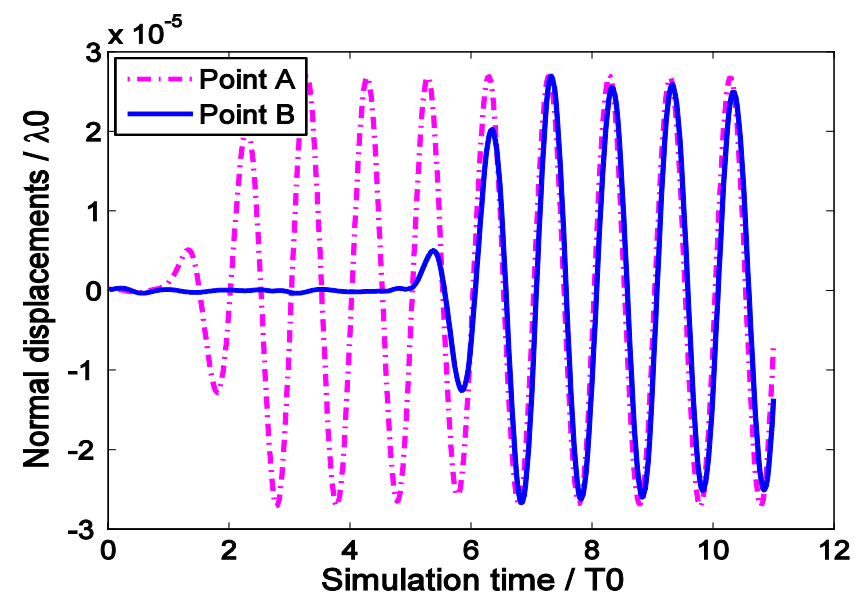

Fig. 3. Displacements in $z$-direction of points A and B.

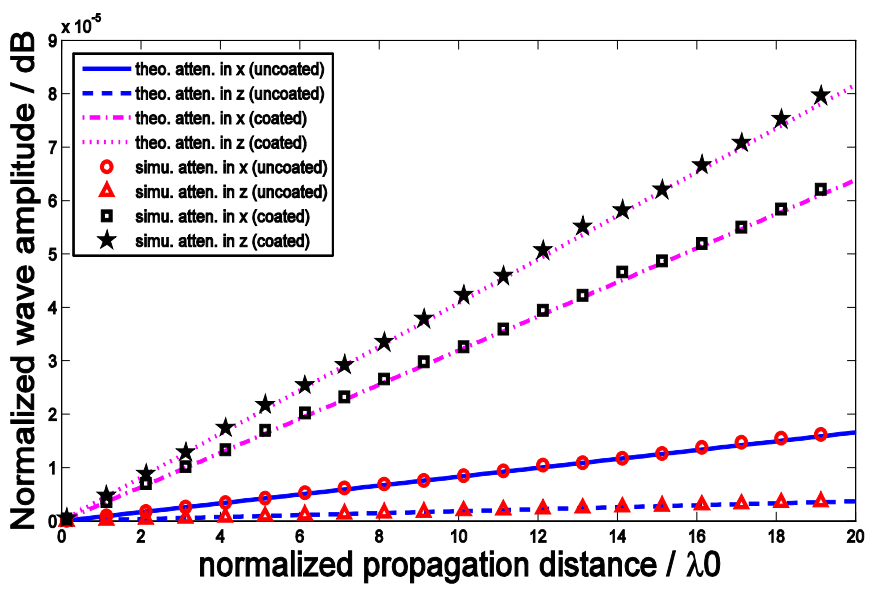

Fig. 4. Wave amplitude versus normalized propagation distance (acoustic attenuation coefficients are proportional to slopes of the lines).

models are demonstrated. In the figure, $-\log \left(u_{\alpha}^{i} / u_{\alpha}^{0}\right)$ versus relative displacements is plotted. $u_{\alpha}^{0}$ indicates displacement in $\alpha$ direction of a reference point. According to the analytical method, the thermoelastic attenuation coefficients in coated condition (upper part) were found to be 13 and $13.3 \mathrm{~dB} / \mathrm{m}$ for $x$-direction and $z$-direction propagation, respectively, whereas those in the uncoated condition (lower part) were 2.7 and $0.6 \mathrm{~dB} / \mathrm{m}$, as represented by the lines. Close values were obtained through the M-FE model, as shown by the scattering symbols.

The proposed M-FE model is straightforward and easy to implement. Through general coupled thermopiezoelectric finite-element approach instead of cumbersome development and excessive simplification in the analytical method, characteristics of the thermoelastic attenuation of the SAW device can be accurately captured. In addition, in modern applications, more complicated geometries are involved in SAW devices, the analytical method becomes no longer applicable. However, though not presented here due to the short of comparable counterparts from the literature (neither analytical nor experimental available to the best of authors knowledge), the proposed method remains an effective approach for simulation.

\section{CONCLUSION}

This paper presents a multi-physics finite-element model dedicating for the thermoelastic damping in SAW devices. The model is validated through comparison with an analytical model in which SAWs of $300 \mathrm{MHz}$ propagate in a lithium niobate substrate under both uncoated and thin-film coated conditions. Numerical values accurately predicted the characteristics of thermoelastically attenuated SAWs. We may conclude that the M-FE method can be employed for thermoelastic attenuation investigation in SAW devices. Compared with other simplified methods, benefits can be drawn because of the simplicity and capacity for handling complicated geometries of the proposed methodology.

\section{REFERENCES}

[1] N. Nam, H. Lim, and S. Shin, "Manipulation of microparticles using surface acoustic wave in microfluidic systems," Korea-Austral. Rheol. J., vol. 23, no. 4, pp. 255-267, Dec. 2011.

[2] K. Lange, B. E. Rapp, and M. Rapp, "Surface acoustic wave biosensors: A review," Anal. Bioanal. Chem., vol. 391, no. 5, pp. 1509-1519, Jul. 2008.

[3] V. Sinha, D. Pathak, A. Kumar, and O. P. Kaushik, "Surface acoustic wave devices for space applications," J. Spacecraft Technol., vol. 18, pp. 61-76, Jul. 2008.

[4] A. P. Mayer, "Thermoelastic attenuation of surface-acoustic waves in coated elastic media," J. Appl. Phys., vol. 68, no. 11, pp. 5913-5915, Dec. 1990.

[5] R. J. Atkin and P. Chadwick, "Surface waves in a heat-conducting elastic body: Correction and extension of a paper of Chadwick and Windle," J. Thermal Stresses, vol. 4, nos. 3-4, pp. 509-521, Mar. 1981.

[6] E. A. Garova, A. I. Kozlov, and V. P. Plesskil, "Theory of the photothermal generation of Rayleigh waves," Soviet Phys. Acoust. New York, vol. 32, pp. 188-192, May 1986.

[7] A. P. Mayer, "Thermoelastic attenuation of surface acoustic waves," Int. J. Eng. Sci., vol. 28, no. 10, pp. 1073-1082, 1990.

[8] X. G. Tian, J. Zhang, Y. P. Shen, and T. J. Lu, "Finite element method for generalized piezothermoelastic problems," Int. J. Solids Struct., vol. 44, nos. 18-19, pp. 6330-6339, Sep. 2007.

[9] A. Goernandt and U. Gabbert, "Finite element analysis of thermopiezoelectric smart structures," Acta Mech., vol. 154, nos. 1-4, pp. 129-140, Dec. 2002.

[10] IEEE Standard on Piezoelectricity, ANSI/IEEE Standard 176-1987, 1987.

[11] D. Karim, S. Ballandras, T. Laroche, and K. Wagner, "Finite element analysis in combination with perfectly matched layer to the numerical modeling of acoustic devices in piezoelectric materials," Appl. Math., vol. 4, pp. 64-71, May 2013.

[12] Y. F. Li, O. B. Matar, V. Preobrazhensky, and P. Pernod, "Convolutionperfectly matched layer (C-PML) absorbing boundary condition for wave propagation in piezoelectric solid," in Proc. IEEE Ultrason. Symp., Nov. 2008, pp. 1568-1571.

[13] Y. Li, "Development of numerical simulation method for nonlinear elastodynamic: Application to acoustic imaging of defect with the help of cavity chaotic transducer," Dept. Eng. Sci., Ecole Centrale de Lille, Villeneuve, France, 2009.

[14] G. Xu, "Direct finite-element analysis of the frequency response of a Y-Z lithium niobate SAW filter," Smart Mater. Struct., vol. 9, no. 6, pp. 937-980, Dec. 2000.

[15] W. P. Mason, Physical Acoustics Principles and Methods, vol. 9. New York, NY, USA: Academic, 1972. 